\title{
28. Precise Control of Polymer Synthesis with a New Catalyst
}

\author{
By Shohei INoue \\ Department of Synthetic Chemistry, Faculty of Engineering, University of Tokyo, \\ Hongo 7-3-1, Bunkyo-ku, Tokyo 113 \\ (Communicated by Seizo Okamura, M. J. A., Oct. 14, 1991)
}

\begin{abstract}
Aluminum porphyrin is an excellent catalyst for the synthesis of polymers from a wide variety of monomers such as epoxide, lactone, and methacrylic ester, to give the polymers with controlled molecular weight. A novel catalyst system composed of aluminum porphyrin and an organoaluminum compound with bulky substituents brings about a remarkably accelerated reaction retaining the controlled molecular weight of the polymer.
\end{abstract}

Key words: Macromolecule; polymerization; porphyrin.

Introduction. The well-defined structure of biopolymers such as protein and nucleic acid, in terms of the molecular weight (number of the repeating units, or degree of polymerization) and the sequence of different repeating units, is essential for their specific functions. The molecular weight and sequence are also important in synthetic polymers in their fundamental properties as well as in practical applications.

The formation of a macromolecule(polymer) takes place by the repeated reactions between the reactive species at the end of the growing polymer molecule and a monomer molecule corresponding to the repeating unit. In the formation of synthetic polymers, it is usually difficult to obtain the polymer with a uniform molecular weight. This is due to possible side reactions of the growing species, which hinder the growing reaction resulting in the non-uniformity of the chain length of polymer molecules. Thus, for the synthesis of polymer with a uniform molecular weight, it is essential to control the reactivity of the growing end of polymer molecule by the selection of an appropriate catalyst or initiator, in such a way that the growth of all polymer molecules proceeds uniformly, without being killed by side reactions (living polymerization).

We have recently found that some metalloporphyrins, particularly of aluminum (e.g.,

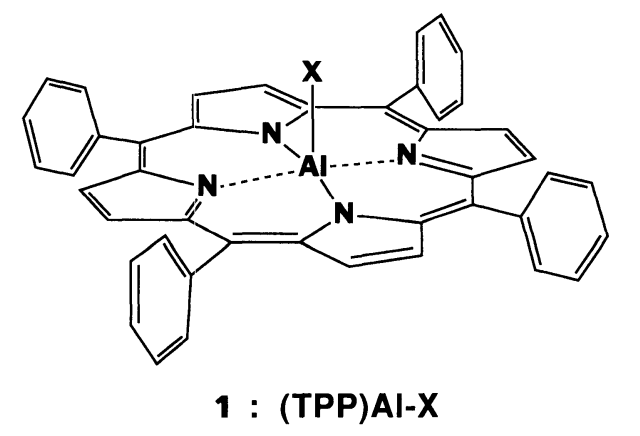

TPP: Tetraphenylporphinato 
(TPP)Al-Cl $+x \underbrace{\mathrm{CH}_{2}-\mathrm{CHMe} \longrightarrow}_{O^{\prime}} \longrightarrow(\mathrm{TPP}) \mathrm{Al}-\mathrm{O}-\mathrm{CHMe}^{-} \mathrm{CH}_{2})_{x} \mathrm{Cl}$

(TPP)Al-Me $+x \mathrm{CH}_{2}=\mathrm{CHMe}-\mathrm{CO}_{2} \mathrm{Me}$

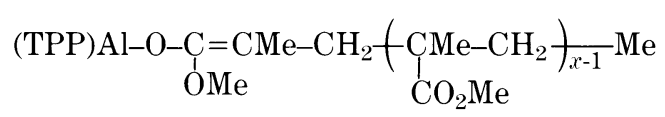

1), are excellent catalyst in this respect, which can be applied to various types of polymerization reaction such as the ring-opening polymerizations of epoxide and lactone, and the addition polymerizations of acrylic monomers. ${ }^{1)}$ Examples are given in equations (1) and (2) for the polymerization of propylene oxide ${ }^{2)}$ and methyl methacrylate, ${ }^{3)}$ respectively. Successful formation of polymers with uniform molecular weight is due to moderate reactivities of the growing species on aluminum porphyrin. On the other hand, the reactions are not satisfactorily fast in some cases. The present report describes a novel excellent catalyst system based on the combination of aluminum porphyrin and an organoaluminum compound with bulky groups, which enables very rapid polymerization reactions giving the polymers with uniform molecular weight.

Principle of design of new catalyst. The reactions of polymer growth such as eqs. 1 and 2 are the attack of the growing species as a nucleophile to the monomer as an electrophile. The reactivity of the monomer is expected to be enhanced for a rapid reaction by the addition of a Lewis acid which can accomodate and activate the monomer by coordination. However, a direct reaction should not occur between the growing species and the Lewis acid which itself is an electrophile.

The principle of designing new catalyst system is to select an appropriate Lewis acid that can coordinate and activate the monomer for nucleophilic attack by the growing species, but does not directly react with the latter. An organoaluminum compound with bulky groups has been found a Lewis acid of this nature in the polymerizations of some vinyl and heterocyclic monomers with aluminum porphyrin (Fig. 1).

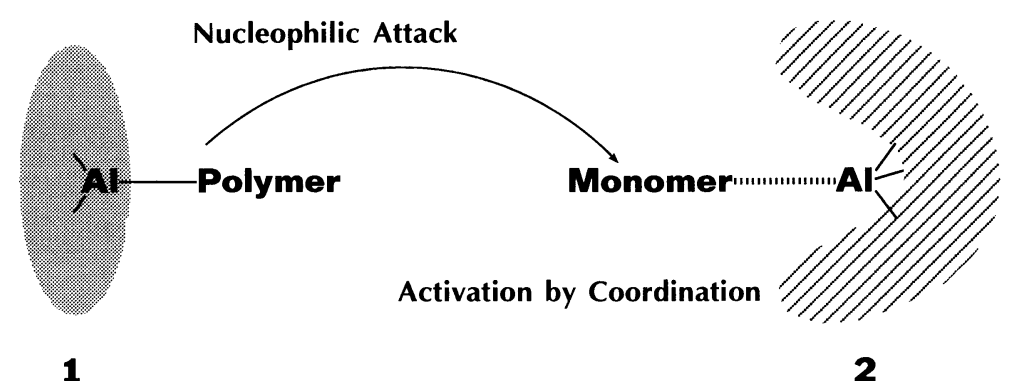

Fig. 1. Concept of New Catalyst Sytem.

Polymerization of methacrylic ester. We have already reported that the aluminum complex of tetraphenylporphyrin with aluminum-methyl group ((TPP)AlMe, $1, \mathrm{X}=\mathrm{CH}_{3}$ ) is an interesting initiator for the living polymerization of methyl methacrylate under irradiation by visible light, with an enolate-aluminum porphyrin as the growing species (eq. 2). However, the reaction is not very fast. For example, when a mixture of (TPP)AlMe(1) and methyl methacrylate (MMA) $\left([\mathrm{MMA}]_{0} /[1]_{0}=217\right)$ in dichoromethane was irradiated at $35^{\circ} \mathrm{C}$ for 2.5 hours in nitrogen atmosphere, the conversion of MMA was $6 \%$. 


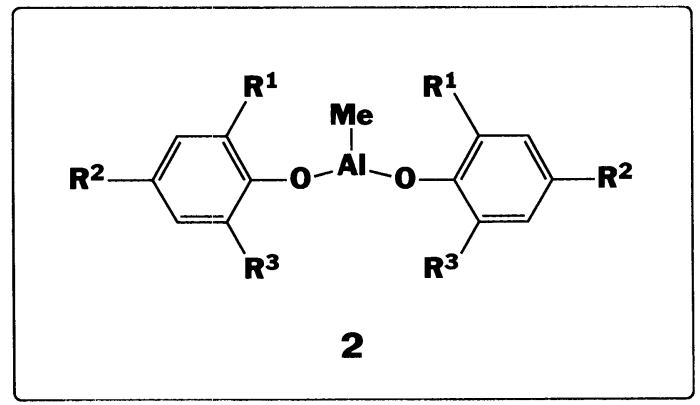

2a: $\mathrm{R}^{1}=\mathrm{R}^{2}=$ tert-butyl, $\mathrm{R}^{3}=\mathrm{H}$

b: $\mathrm{R}^{1}=\mathrm{R}^{2}=\mathrm{R}^{3}=$ tert-butyl

The polymer formed had $\overline{\mathrm{M}} n=1600$ and $\mathrm{Mw} / \mathrm{Mn}=1.13$, where $\overline{\mathrm{M}} \mathrm{n}$ and $\overline{\mathrm{M}} \mathrm{w}$ represent the number-average and weight-average molecular weights, respectively. The value of $\overline{\mathrm{M}} \mathrm{w} / \overline{\mathrm{Mn}}$ close to unity indicates the narrow molecular weight distribution (uniform molecular weight).

Surprisingly, when a methylaluminum diphenoxide with bulky substituents 2a was added at this moment to the reaction mixture $\left([2]_{0} /[1]_{0}=3.0\right)$, the polymerization proceeded very rapidly with heat evolution, and was completed in only 3 seconds. The molecular weight $\overline{\mathrm{M}}$ n of the polymer increased to 25500 retaining the narrow distribution ( $\overline{\mathrm{M} w} / \overline{\mathrm{M}} \mathrm{n}=1.07$ ), as evidenced by gel permeation chromatography of the reaction mixture (Fig. 2). The extent of acceleration is as high as about 46000 times.

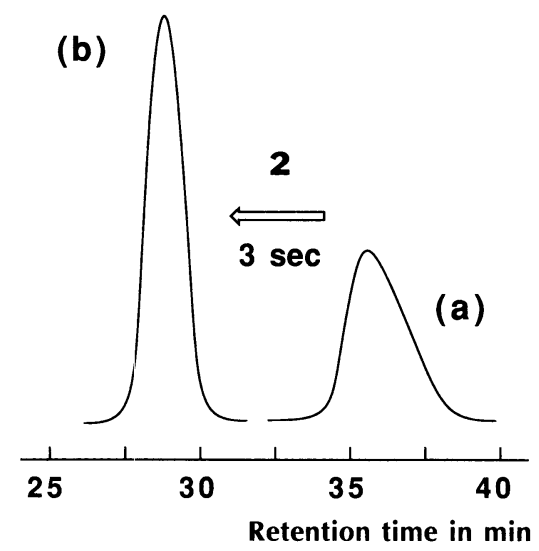

Fig. 2. Gel permeation chromatogram of the polymerization system of MMA with 1(a), followed by the addition of 2(b). For the reaction conditions, see text. Shorter retention time corresponds to higher molecular weight.

The molecular weight of the polymer could be controlled by changing the ratio of the monomer to aluminum porphyrin. On the other hand, when the ratio of the aluminum phenoxide 2 to aluminum porphyrin 1 was varied, the rate of reaction was found to change, while the number of polymer molecules, as calculated from the molecular weight and the conversion, remained equal to that of aluminum porphyrin molecules. 2 itself could not bring about the polymerization of MMA.

Therefore, $\mathbf{2}$ is considered to play a role of catalyst, as a Lewis acid to coordinate and 
activate the monomer, the reactivity of which is enhanced for the nucleophilic attack of the growing species on aluminum porphyrin, resulting in the very remarkable acceleration. On the other hand, direct reaction between the growing species on porphyrin and 2 is suppressed by the virtue of the bulkiness of these species.

Polymerization of epoxide. Methylaluminum phenoxide with bulky substituents 2 was found also effective for the acceleration of the living polymerization of epoxide initiated with aluminum porphyrin. For example, the polymerization of propylene oxide (PO) initiated with (TPP) $\mathrm{AlCl}(1, \mathrm{x}=\mathrm{Cl})(\mathrm{eq} .1)\left([\mathrm{PO}]_{0} /[1]_{0}=200\right)$ in dichloromethane at room temperature proceeded to $19.8 \%$ conversion after 7 hours to give the polymer with $\mathrm{Mn}=3000, \mathrm{Mw} / \mathrm{Mm}=1.05$. Upon addition of $2 \mathrm{~b}\left([2]_{0} /\left[\mathbf{1}_{0}\right]=0.5\right)$ at this moment, the reaction with heat evolution ensued to reach $85.5 \%$ conversion in 3 minutes to form the polymer with $\overline{\mathrm{M}} \mathrm{n}=11900, \overline{\mathrm{M} w} / \overline{\mathrm{M}} \mathrm{n}=1.21$. The acceleration corresponds to about 500 times. In this case as well, the molecular weight of the polymer could be controlled by the ratio of monomer to aluminum porphyrin.

Further development of the new catalyst system is in progress in order to extend the scope to other types of monomers and for the application to molecular design of polymers with well-defined structure.

\section{References}

1) Inoue, S.: Frontiers of Macromolecular Science (eds. T. Saegusa, T. Higashimura and A. Abe). Blackwell, p. 73 (1989).

2) Aida, T., and Inoue, S.: Macromolecules, 14, 1162 (1981).

3) Kuroki, M., Aida, T., and Inoue, S.: J. Am. Chem. Soc., 109, 4737 (1987). 